\title{
Identification of DNA methylation markers for early detection of CRC indicates a role for nervous system-related genes in CRC
}

\author{
Glenn Rademakers ${ }^{1 \dagger}$, Maartje Massen ${ }^{1 \dagger}$, Alexander Koch ${ }^{1 \dagger}$, Muriel X. Draht ${ }^{1}$, Nikkie Buekers ${ }^{1}$, \\ Kim A. D. Wouters ${ }^{1}$, Nathalie Vaes ${ }^{1}$, Tim De Meyer ${ }^{2}$, Beatriz Carvalho ${ }^{3}$, Gerrit A. Meijer ${ }^{3}$, James G. Herman ${ }^{4}$, \\ Kim M. Smits ${ }^{1}$, Manon van Engeland ${ }^{1}$ and Veerle Melotte ${ }^{1,5^{*}}$ (D)
}

\begin{abstract}
Purpose: Colonoscopy and the fecal immunochemical test (FIT) are currently the most widely used screening modalities for colorectal cancer (CRC), however, both with their own limitations. Here we aim to identify and validate stool-based DNA methylation markers for the early detection of CRC and investigate the biological pathways prone to DNA methylation.

Methods: DNA methylation marker discovery was performed using The Cancer Genome Atlas (TCGA) colon adenocarcinoma data set consisting of normal and primary colon adenocarcinoma tissue. The performance of the five best candidate markers and a previously identified marker, NDRG4, was evaluated on tissues and whole stool samples of healthy subjects and CRC patients using quantitative MSP assays. The results were compared and combined with FIT data. Finally, pathway and gene ontology enrichment analyses were performed using ToppFun, GOrilla and clusterProfiler.

Results: GDNF, HAND2, SLC35F3, SNAP91 and SORCS1 were ranked as the best performing markers. Gene combinations of all five markers, NDRG4 and FIT were evaluated to establish the biomarker panel with the highest diagnostic potential, resulting in the identification of GDNF/SNAP91/NDRG4/FIT as the best performing marker panel. Pathway and gene ontology enrichment analyses revealed that genes associated with the nervous system were enriched in the set of best performing CRC-specific biomarkers.

Conclusion: In silico discovery analysis using TCGA-derived data yielded a novel DNA-methylation-based assay for the early detection of CRC, potentially improving current screening modalities. Additionally, nervous system-related pathways were enriched in the identified genes, indicating an epigenetic regulation of neuronal genes in CRC.
\end{abstract}

Keywords: Colorectal cancer, Diagnostic markers, In silico discovery, DNA methylation, Nervous system

*Correspondence: veerle.melotte@maastrichtuniversity.nl

${ }^{\dagger}$ Glenn Rademakers, Maartje Massen and Alexander Koch have

contributed equally to this work

${ }^{1}$ Department of Pathology, GROW - School for Oncology

and Developmental Biology, Maastricht University Medical Center, P.O. Box 616, 6200 MD Maastricht, The Netherlands

Full list of author information is available at the end of the article

\section{Introduction}

Colorectal cancer (CRC) is the third most common type of cancer and the second cause of cancer-related mortality worldwide [1]. Implementing screening programs for early CRC detection leads to substantial reductions in CRC incidence and mortality [2]. Colonoscopy and the fecal immunochemical test (FIT) are the most widely used modalities for CRC screening [3, 4], but both have original author(s) and the source, provide a link to the Creative Commons licence, and indicate if changes were made. The images or other third party material in this article are included in the article's Creative Commons licence, unless indicated otherwise in a credit line to the material. If material is not included in the article's Creative Commons licence and your intended use is not permitted by statutory regulation or exceeds the permitted use, you will need to obtain permission directly from the copyright holder. To view a copy of this licence, visit http://creativecommons.org/licenses/by/4.0/. The Creative Commons Public Domain Dedication waiver (http://creativeco mmons.org/publicdomain/zero/1.0/) applies to the data made available in this article, unless otherwise stated in a credit line to the data. 
limitations. The invasive nature of colonoscopy is associated with risks of bleeding, bowel perforation, low participation rates and high costs, while the sensitivity and specificity of FIT are suboptimal $[5,6]$. To improve the current FIT, alterations in CRC-derived DNA found in bodily fluids (e.g., blood and feces) have been evaluated [7]. Three DNA methylation markers (SEPTIN9, NDRG4 and $B M P 3$ ) have been incorporated in FDA-approved screening tests for early CRC detection [8]. Two assays detect SEPTIN9 methylation in blood (Epi proColon ${ }^{\circledR} 2.0$ $\mathrm{CE}$ and Colovantage ${ }^{\circledR}$ ) with a sensitivity of $58.6-81.0 \%$ and a specificity of $80.0-99.0 \%$ [9-18]. The multitarget stool DNA test Cologuard $^{\circledR}$, combining KRAS mutations, BMP3 and NDRG4 methylation and an immunochemical assay for human hemoglobin $[19,20]$, reported a sensitivity of $92.3 \%$ and specificity of $86.6 \%[19,21]$.

The observation that only $0.8 \%$ of the reported DNA methylation markers have been translated into a commercial product illustrates the complexity of translating laboratory discoveries to clinical applications [8]. Reasons for this include flawed study designs, suboptimal marker identification and methodological issues [8]. To improve clinical translation, we previously recommended (1) to critically evaluate the genomic location of DNA methylation biomarkers and (2) to use publicly available (epi)genomics databases [8].

Here, we applied these recommendations to optimize the implementation of early CRC detection DNA methylation biomarkers using publicly available data from The Cancer Genome Atlas (TCGA) [22]. Five candidate DNA methylation biomarkers were identified that were further validated in CRC tissue and stool samples. Many of the identified candidate markers were involved in nervous system-related pathways, indicating a role for the nervous system in colorectal carcinogenesis.

\section{Methods}

For more detailed information, see supplemental methods.

\section{Gene discovery analysis}

The following TCGA data sets for colon adenocarcinoma were used: clinical patient data, level 3 Infinium $450 \mathrm{k}$ DNA methylation data and level 3 Illumina HiSeq RNAseq V2 gene expression data (upper quartile normalized RSEM expression estimates). Methylation and gene expression data were available for 12,263 genes.

TCGA methylation data were selected for the Infinium $450 \mathrm{k}$ probes located in promoter $\mathrm{CpG}$ islands (i.e., the region from $-1000 \mathrm{bp}$ to $+500 \mathrm{bp}$ around the transcription start site) that were unmethylated in normal samples (median $\beta$ over all normal samples $<0.20$ ) (Fig. 1, "Introduction" section). Because a clinically relevant early detection marker needs to identify all tumor stages, we compared normal samples $(n=37)$ with stage I and II tumor samples $(n=146)$ and stage III and IV tumor samples $(n=116)$ using a one-sided Mann-Whitney test ( $\mu=-0.25)$. Resulting $P$ values were corrected using false discovery rate (FDR) correction and only probes with an $\mathrm{FDR}<0.05$ and a difference in median $\beta<-0.25$ were retained (Fig. 1, "Methods" section). Probes for which at least one other differentially methylated probe was located within $750 \mathrm{bp}$ up or downstream were selected. Next, downregulated genes in the tumor samples were identified in the RNA-seq expression data (normal tissue: $n=41$, primary tumor tissue: $n=285$ ) with the one-sided Mann-Whitney test $(\mu=1)$, using an FDR cutoff of 0.05 (Fig. 1, "Results" section). The results of both differential methylation analyses were combined with the results of the differential gene expression analysis yielding a list of 236 genes (Fig. 1, "Discussion" section) [8].

To ensure tumor-specificity of the methylation, we evaluated methylation of the selected probes in normal samples from fourteen TCGA cancer types, i.e., breast cancer, colon adenocarcinoma, lung squamous cell carcinoma, prostate adenocarcinoma, head and neck squamous cell carcinoma, lung adenocarcinoma, bladder urothelial carcinoma, kidney renal clear cell carcinoma, kidney renal papillary cell carcinoma, liver hepatocellular carcinoma, thyroid carcinoma, uterine corpus endometrial carcinoma, colorectal adenocarcinoma and esophageal carcinoma. For every probe and tissue type, the percentage of methylated (median $\beta>0.20$ ) samples was calculated. Only probes for which the maximal fraction of methylated samples was lower than $25 \%$ in each tissue type were retained (Fig. 1, Sect. 5), revealing 221 genes. All analyses were performed using the $\mathrm{R}$ programming language (version 3.2.3).

\section{Tissue samples}

Formalin-fixed, paraffin-embedded tumor tissue (CRC) and matching normal tissue was retrospectively collected from the tissue archives of the Pathology Department of the Maastricht University Medical Center (MUMC) for 34 patients diagnosed between 1995 and 2003 (Additional file 1: Table S1). This study was approved by the Medical Ethical Committee of the MUMC (MPTC 2015-12).

\section{Collection of FIT and stool samples}

Stool samples from colonoscopy-negative controls $(n=50)$ were prospectively obtained from a workplacebased CRC screening study at MUMC. Stool samples from CRC 121 patients $(n=43)$ were prospectively collected in the noninvasive markers for CRC (NIM) study at MUMC. Both studies were approved by the Medical 


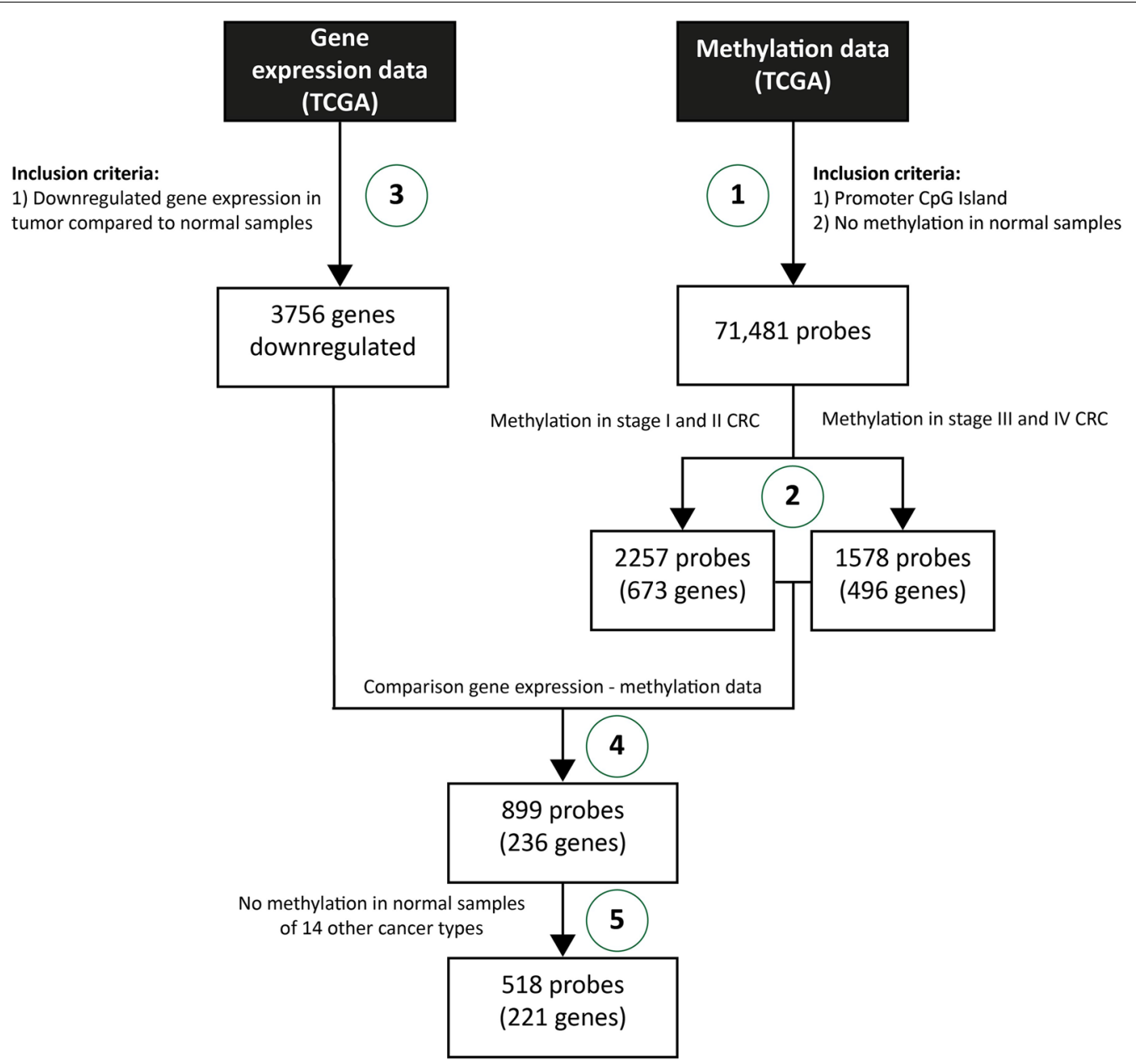

Fig. 1 Pipeline to select candidate methylation markers using The Cancer Genome Atlas (TCGA) database. Marker discovery is based on a selection procedure using methylation data (right section) and gene expression data (left section). The DNA methylation analysis resulted in a list of Infinium $450 \mathrm{k}$ probes that were: (1) located in promoter CpG islands, (2) unmethylated in normal colon tissue, and (3) hypermethylated in tumor samples over all four stages cancer development. This list was compared with a list of genes downregulated in tumor compared to normal samples, and we checked the methylation status of the remaining probes in normal samples from 14 different cancer types, resulting in a list of 221 genes. Finally, we designed and tested primers for the probes with the highest sensitivity and specificity based on the TCGA data, resulting in the top five potential early detection markers for CRC

Ethical Committee of the MUMC (METC 04-088, METC 08-2-038) and written informed consent was obtained from all patients. Population characteristics are shown in Table 1.

Participants collected one bowel movement in stool collection container (Exact Sciences) just before the bowel preparation for the colonoscopy. Directly after, stabilization buffer (Exact Sciences) was added. Simultaneously, FIT (OC-Sensor, Eiken Chemical, Tokyo, Japan) was performed, according to the manufacturer's instructions. A quantitative concentration of $50 \mathrm{ng} \mathrm{Hb} / \mathrm{ml}$ test buffer was set as threshold for a positive FIT. The FIT and stool samples were delivered to the laboratory within $72 \mathrm{~h}$ after collection, stored at $-20{ }^{\circ} \mathrm{C}$ and further processed as previously described [23].

\section{Methylation data and statistical analyses}

Methylation frequencies of the selected genes were determined in carcinoma and matched normal tissues and compared with McNemar's test. A receiver operating characteristic (ROC) curve analysis and area under the curve (AUC) were established to assess their diagnostic utility. For the AUC, a 95\%-confidence interval was estimated using a nonparametric method. The qMSP cutoff value for each marker was determined based on 
Table 1 Clinicopathological features of the stool samples obtained from a hospital-based series

\begin{tabular}{|c|c|c|}
\hline Patient demographics & Normal $(N=50|n(\%)|)$ & $\begin{array}{l}\text { Carcinoma } \\
(\mathrm{N}=42 \mid n \\
(\%) \mid)\end{array}$ \\
\hline \multicolumn{3}{|l|}{ Sex } \\
\hline Male & $24(48.0)$ & $28(66.7)$ \\
\hline Female & $26(52.0)$ & $14(33.3)$ \\
\hline \multicolumn{3}{|l|}{ Age (years) } \\
\hline Median ( \pm StDev) & $55.5( \pm 3.7)$ & $70.5( \pm 10.5)$ \\
\hline \multicolumn{3}{|l|}{ Histological type } \\
\hline Adenocarcinoma & - & $31(73.8)$ \\
\hline Signet ring cell carcinoma & - & $1(2.4)$ \\
\hline Mucinous adenocarcinoma & - & $2(4.8)$ \\
\hline High-grade neuroendocrine carcinoma & - & $1(2.4)$ \\
\hline Unknown & & $7(16.6 \%)$ \\
\hline \multicolumn{3}{|l|}{ Differentiation grade } \\
\hline Poor & - & $6(14.3)$ \\
\hline Moderate/well & - & $25(59.5)$ \\
\hline Unknown & - & $11(26.2)$ \\
\hline \multicolumn{3}{|l|}{ T-stage } \\
\hline Stage 1 & - & $0(0.0)$ \\
\hline Stage 2 & - & $7(16.7)$ \\
\hline Stage 3 & - & $24(57.1)$ \\
\hline Stage 4 & - & $10(23.8)$ \\
\hline Unknown & - & $1(2.4)$ \\
\hline \multicolumn{3}{|l|}{ Location } \\
\hline Proximal & - & $14(33.3)$ \\
\hline Distal & - & $25(59.5)$ \\
\hline Unknown/other & - & $3(7.2)$ \\
\hline
\end{tabular}

Tissue retrieved retrospectively from the tissue archive of the department of Pathology of the Maastricht University Medical Center

${ }^{ \pm} P<0.000$ compared to normal, one-way ANOVA was used

the highest likelihood ratio (see Additional file 1: Supplementary methods). Promoter methylation was considered positive if the methylation value was higher than the predetermined cutoff. Next, the best performing marker panel was identified. The Pearson chi-square test was used to compare methylation frequencies in fecal DNA between CRC patients and healthy subjects. All statistical analyses were performed using IBM SPSS Statistics 23, $R$ programming language (version 3.2.3) or Graphpad Prism (version 5.03).

\section{Gene ontology and pathway analyses}

Gene ontology enrichment analyses using Gorilla [24] and clusterProfiler [25] were performed on the 221 differentially methylated and downregulated genes compared to the background gene set (12, 263 genes). Gene ontology analyses were performed on three different subsets of gene ontologies: cellular component, molecular function and biological process. The number of nervous system-related gene ontologies in the enriched sets was compared with the frequency of these ontologies in the complete set of ontologies (Gene Ontology Consortium http://geneontology.org/, link to the ontology file: http://purl.obolibrary.org/obo/go/go-basic. obo). To identify the number of neuronal-related gene ontologies, we used neuronal-related keywords ('neuro,' 'neuron,' 'neuronal,' 'neural,' 'nervous,' 'axon,' 'dendritic, 'synaptic,' 'synapse, 'learning,' 'memory, 'brain,' 'hippocampus') and applied the Fisher's Exact test to compare the frequencies of the neuronal-related ontologies ( $\mathrm{R}$ version 3.2.3).

In addition, pathway analysis was performed using three major pathway databases (Reactome, KEGG and PantherDB) using the ToppFun application (http:// toppgene.cchmc.org) [26]. Pathways identified with a cutoff value of $P<0.05$ were considered for further analysis. As standard methods to correct $P$ values for multiple testing tend to be conservative, these corrections were not applied. 


\section{Results}

In silico identification of early CRC detection DNA methylation markers using publicly available TCGA data A multistep in silico gene discovery analysis was used to identify novel candidate DNA methylation markers for early CRC detection (Fig. 1). The list of Infinium Human Methylation $450 \mathrm{~K}$ BeadChip probes $(n=485,577)$ was initially reduced to 71,481 probes based on location in promoter $\mathrm{CpG}$ islands and the absence of methylation in normal samples. Based on their methylation status, 2257 probes/673 genes in stage I/II and 1578 probes/496 genes in stage III/IV were identified. This list was combined with 3756 genes identified as downregulated in tumor compared to normal samples, resulting in 899 probes/236 genes. Finally, to select tumor specific probes, all 899 probes were investigated in normal samples of fourteen other cancer types, resulting in 518 probes/221 genes (Fig. 1). The top twenty genes with the highest sensitivity and $100 \%$ specificity were selected for further investigation.

\section{Methylation frequencies of the best performing genes in CRC tissue}

After performing MSPs on 34 matched CRC and normal colon tissue samples, we obtained MSP data for ten genes (dropouts due to unsuccessful and suboptimal MSP primer design). We identified GDNF, HAND2, SLC35F3, SNAP91 and SORCS1 as the five best performing tissue candidates and selected these markers for further qMSP analysis. Methylation frequencies for these genes differed between CRC and normal tissue $(P<0.0001$ for all genes) with high methylation in CRC; SORCS1 (91.2\%), SLC35F3 (88.2\%), SNAP91 (85.3\%), GDNF (76.5\%) and HAND2 (73.5\%) (Additional file 2: Figure S1). In the matched normal tissue samples, methylation was lower; HAND2 (8.8\%), SNAP91 and SORCS1 (both 5.9\%), and GDNF and SLC35F3 (both 2.9\%) \%) (Additional file 2: Figure S1). This in situ validation of in silico identified DNA methylation markers shows the potential of public data for biomarker discovery.

\section{Sensitivity and specificity for the best performing genes in fecal DNA of CRC patients}

The diagnostic performance of the five identified DNA methylation markers in fecal DNA was assessed in stool samples from 50 healthy subjects and 43 CRC patients. The AUCs for GDNF, HAND2, SLC35F3, SNAP91 and SORCS1 in stool were 0.726 [95\%-CI 0.619-0.834], 0.722 [95\%-CI 0.615-0.829], 0.736 [95\%-CI 0.632-0.841], 0.799 [95\%-CI 0.704-0.893] and 0.707 [95\%-CI 0.597-0.817], respectively (Fig. 2a). For all markers, the highest likelihood ratio was observed at $98.0 \%$ specificity. Using this fixed specificity of $98.0 \%$, SNAP91 had the highest sensitivity (46.5\% [95\%-CI 31.2-62.4\%], cutoff $=112.6$ copies/ $\mu \mathrm{l})$ followed by GDNF (sensitivity: 41.9\% [95\%-CI 27.0$57.9 \%$, cutoff $=21.16 \mathrm{copies} / \mu \mathrm{l}$ ) and SORCS1 (sensitivity: $41.9 \%$ [95\%-CI 27.0-57.9\%], cutoff $=110.0$ copies/ $\mu \mathrm{l}$ ), SLC35F3 (sensitivity: 39.5\% [95\%-CI 25.0-55.6\%], cutoff $=63.82 \mathrm{copies} / \mu \mathrm{l}$ ) and HAND2 (sensitivity: $32.6 \%$ [95\%-CI 19.1-48.5\%], cutoff $=79.02$ copies/ $\mu$ l) (Fig. 2b). The association between the methylation status and clinicopathological features is shown in Table 2.

To determine the best diagnostic panel, all possible marker combinations were analyzed. The panel detecting GDNF or SNAP91 methylation was the optimal panel with $48.8 \%$ sensitivity [95\%-CI 33.9-63.7\%] and 98.0\% specificity (AUC 0.727 [95\%-CI 0.621-0.834], Fig. 2c, d). Without predetermined cutoffs, a panel of SLC35F3 and SNAP91 appeared the best performing marker panel (sensitivity 65.1\%, specificity 82.0\%, AUC 0.827 [95\%CI 0.701-0.954], Fig. 2e, f). Other marker combinations were observed with higher sensitivities (67.4-81.4\%), however, but with reduced specificities (65.3-81.6\%).

\section{The performance of the in silico identified DNA methylation markers and NDRG4 in combination with FIT}

To determine the added diagnostic value to currently applied diagnostic assays, the marker panel was combined with our previously identified and established methylation marker NDRG4 [27] and the FIT.

Adding NDRG4 methylation (Fig. 3a, b) to the panel leads to a slightly higher AUC (0.745; [95\%-CI 0.624$0.867]$ ) and sensitivity (51.2\% [95\%-CI 36.3-66.1\%]) without changes in specificity (98.0\%), as compared to GDNF and SNAP91 methylation alone (Additional file 3: Figure $\mathrm{S} 2 \mathrm{a}, \mathrm{S} 2 \mathrm{~b})$. No other panel outperformed the GDNF/ SNAP91/NDRG4 panel.

Using a cutoff of $50 \mathrm{ng} \mathrm{Hb} / \mathrm{ml}$ buffer, FIT sensitivity for CRC detection was higher than any of the described single markers or marker panels alone; sensitivity $81.4 \%$ [95\%-CI 69.8-93.0\%] at a specificity of 98.0\%, AUC 0.946 (Fig. 3c, d). Combinations of single markers and FIT showed higher sensitivities (86.0\% [95\%-CI 75.7-96.4\%] for GDNF, SNAP91 and SORCS1, and 83.7\% [95\%-CI 72.7-94.8\%] for SLC35F3 but with a lower specificity (96.0\%) (Additional file 3: Figure S2c).

When combining the best performing marker panel (GDNF/SNAP91/NDRG4) with FIT, the AUC reached 0.940 and the sensitivity increased to $86.0 \%$ [95\%-CI 75.6-96.4\%] at 96.0\% specificity (Fig. 3d, e), which is higher compared to the marker panel alone (37\% increase) and slightly higher than the FIT alone (4.6\% increase). Interestingly, the panel combining SNAP91/NDRG4/FIT achieved the same performance 


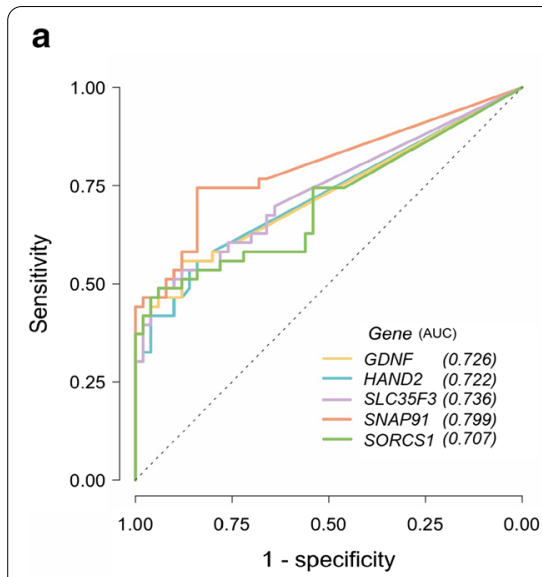

C

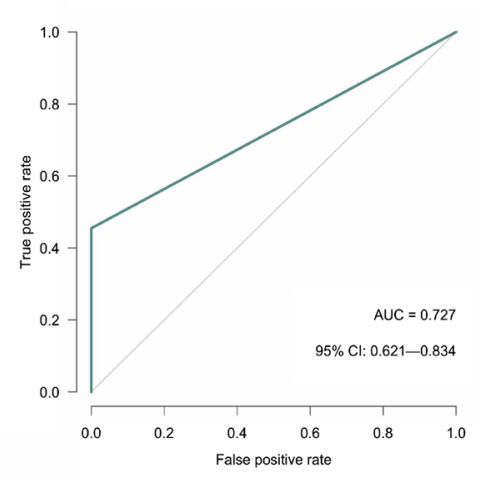

e

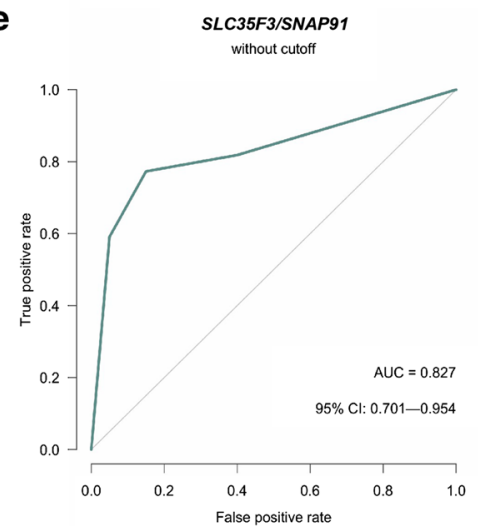

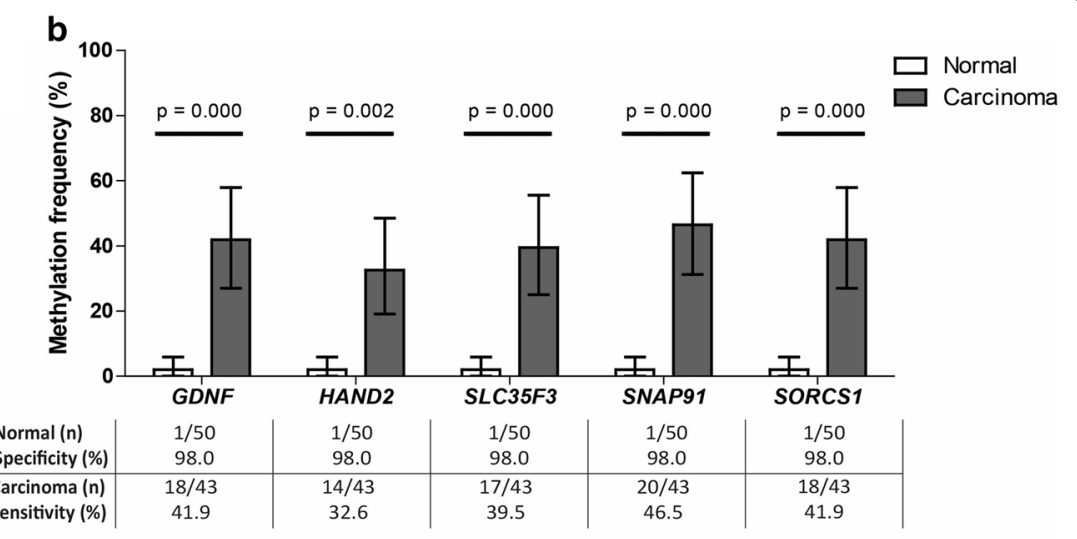

d
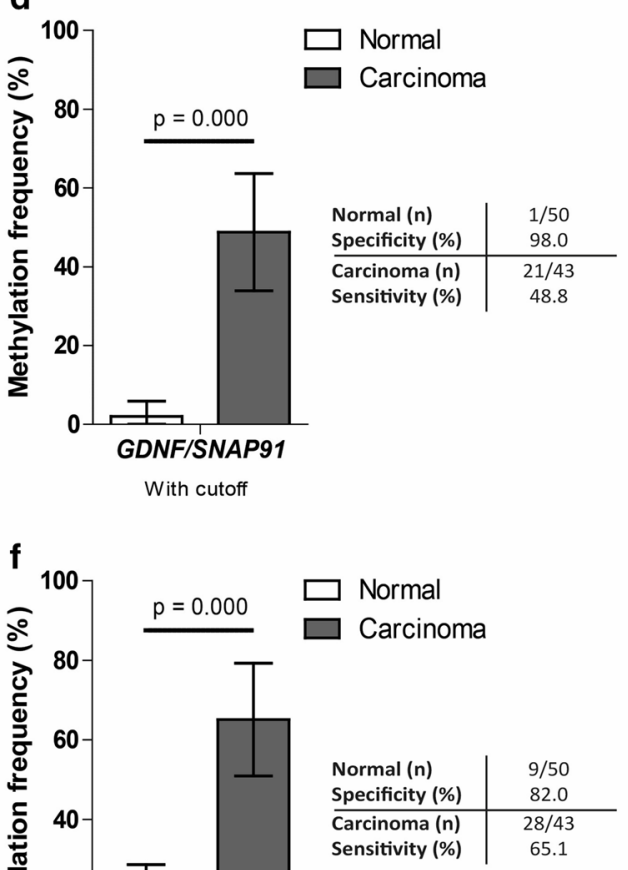

Fig. 2 Early detection methylation marker validation using fecal DNA from healthy controls and CRC patients. a Receiver operating characteristic (ROC) curve for marker validation of GDNF, HAND2, SLC35F3, SNAP91 and SORCS1 on fecal DNA to determine optimal sensitivity/specificity. The jagged lines indicate the different ROC curves for each independent marker. The dashed line represents the line of no discrimination between good and bad classification. b Methylation frequency (\%) of single markers in fecal DNA of healthy controls (white bar) and CRC patients (black bar). The exact number of methylated samples is indicated in the table below for both groups (methylated samples/total number samples). For the healthy control group, the specificity is given; for carcinomas the sensitivity was determined. Pearson's chi-square test was used to calculate $P$ values. c ROC curve for the best performing marker panel (GDNF/SNAP91) based on the cutoffs for GDNF and SNAP91. The jagged (green) line indicates the ROC curve for this specific panel. The gray line represents the line of no discrimination between good and bad classification. $\mathbf{d}$ Methylation frequency of the GDNF/SNAP91 marker panel in fecal DNA of healthy controls (white bar) and carcinomas (dark gray bar). Fisher's exact test was used to calculate $P$ values. e ROC curve for the best performing marker panel (SLC35F3/SNAP91) without cutoff. f Methylation frequency of the SLC35F3/SNAP91 marker panel in fecal DNA of healthy controls (white bar) and carcinomas (dark gray bar). Fisher's exact test was used to calculate $P$ values 
Table 2 Promoter methylation markers in colorectal carcinomas compared with clinicopathological features

\begin{tabular}{|c|c|c|c|c|c|c|c|c|c|c|}
\hline & \multicolumn{2}{|l|}{ GDNF } & \multicolumn{2}{|l|}{ HAND2 } & \multicolumn{2}{|l|}{ SLC35F3 } & \multicolumn{2}{|l|}{ SNAP91 } & \multicolumn{2}{|l|}{ SORCS1 } \\
\hline & M & U & M & U & M & U & M & U & M & U \\
\hline & $n(\%)$ & $n(\%)$ & $n(\%)$ & $n(\%)$ & $n(\%)$ & $n(\%)$ & $n(\%)$ & $n(\%)$ & $n(\%)$ & $n(\%)$ \\
\hline \multicolumn{11}{|l|}{ Tumors } \\
\hline Number & $17(40.5)$ & $25(59.5)$ & $11(26.2)$ & $31(73.8)$ & $15(35.7)$ & $27(64.3)$ & $19(45.2)$ & $23(54.8)$ & $17(40.5)$ & $25(59.5)$ \\
\hline \multicolumn{11}{|l|}{ Sex } \\
\hline Male & $11(64.7)$ & $17(68.0)$ & $8(72.7)$ & $20(64.5)$ & $10(66.7)$ & $18(66.7)$ & $11(57.9)$ & $17(73.9)$ & $10(58.9)$ & 18 (72.0) \\
\hline Female & $6(35.3)$ & $8(32.0)$ & $3(27.3)$ & $11(35.5)$ & $5(33.3)$ & $9(33.3)$ & $8(42.1)$ & $6(26.1)$ & $7(41.1)$ & $7(28.0)$ \\
\hline$P$ value & 0.824 & 0.620 & 1.000 & 0.273 & 0.374 & & & & & \\
\hline \multicolumn{11}{|l|}{ Cancer stage } \\
\hline Stage I & $0(0.0)$ & $0(0.0)$ & $0(0.0)$ & $0(0.0)$ & $0(0.0)$ & $0(0.0)$ & $0(0.0)$ & $0(0.0)$ & $0(0.0)$ & $0(0.0)$ \\
\hline Stage II & $5(31.3)$ & $2(8.0)$ & $2(20.0)$ & $5(16.1)$ & $3(21.4)$ & $4(14.8)$ & $6(33.3)$ & $1(4.3)$ & $4(25.0)$ & $3(12.0)$ \\
\hline Stage III & $8(50.0)$ & $16(64.0)$ & $5(50.0)$ & $19(61.3)$ & $7(50.0)$ & $17(63.0)$ & $8(44.5)$ & $16(69.6)$ & $8(50.0)$ & $16(64.0)$ \\
\hline Stage IV & $3(18.7)$ & $7(28.0)$ & $3(30.0)$ & $7(22.6)$ & $4(28.6)$ & $6(22.2)$ & $4(22.2)$ & $6(26.1)$ & $4(25.0)$ & $6(24.0)$ \\
\hline$P$ value & 0.153 & 0.818 & 0.721 & 0.047 & 0.523 & & & & & \\
\hline \multicolumn{11}{|l|}{ Tumor location } \\
\hline Proximal & $0(0.0)$ & 14 (60.9) & $1(9.1)$ & $13(46.4)$ & $0(0.0)$ & $14(56.0)$ & $0(0.0)$ & $14(66.7)$ & $0(0.0)$ & $14(60.9)$ \\
\hline Distal & $16(100.0)$ & $9(39.1)$ & $10(90.9)$ & 15 (53.6) & $14(100.0)$ & $11(44.0)$ & $18(100.0)$ & $7(33.3)$ & $16(100.0)$ & $9(39.1)$ \\
\hline$P$ value & 0.000 & 0.029 & 0.000 & 0.000 & 0.000 & & & & & \\
\hline \multicolumn{11}{|l|}{ Age (years) } \\
\hline Mean age $( \pm S D)$ & $71( \pm 7.6)$ & $70( \pm 12.2)$ & $73( \pm 7.7)$ & $70( \pm 11.2)$ & $70( \pm 9.6)$ & $71( \pm 11.1)$ & $70( \pm 9.0)$ & $71( \pm 11.7)$ & $70( \pm 9.0)$ & $71( \pm 11.5)$ \\
\hline$P$ value & 0.927 & 0.333 & 0.812 & 0.663 & 0.885 & & & & & \\
\hline
\end{tabular}

Pearson's chi-square (sex, stage and tumor location) and independent samples $t$ tests (age) were used to calculate $P$ values

(Fig. 3f) with no other panels outperforming this combination (Additional file 1: Table S2).

\section{Gene ontology enrichment and pathway analysis of DNA methylation markers for CRC}

When investigating gene functions, we observed that twelve out of the top twenty (60.0\%) genes were related to the nervous system based on their gene ontologies (Additional file 1: Table S3), resembling the previously identified neuronal-specific expression of NDRG4 [27-29].

Gene ontology enrichment analyses were performed to investigate whether neuronal-related gene ontologies were also enriched in the 221 genes (Fig. 1). Using GOrilla, 46 biological process ontologies were found to be enriched, including seventeen neuronal-related ontologies (37.0\%). Using clusterProfiler, 20/32 (62.5\%) of the enriched ontologies were neuronal-related. Additionally, multiple neuronal-related cellular component ontologies were found to be enriched: 12/23 (52.2\%) and $8 / 10(80.0 \%)$, respectively. No neuronal-related molecular function ontologies were enriched in our target gene set. To put these numbers into context, we compared the frequency of neuronal gene ontologies between our lists of enriched gene ontologies and the complete set of all 47,133 ontologies. For the biological process ontologies,
$4.7 \%$ of all ontologies were nervous system related in the complete set compared to $37.0 \%$ (GOrilla) and 62.5\% (clusterProfiler) in the enriched set (Fig. 4a). Similar to the biological process ontologies, the frequency of neuronal-related cellular component ontologies was increased in the enriched set $(52.2-80.0 \%)$ as only $5.5 \%$ of all ontologies were neuronal-related in the complete set (Fig. 4a).

Using the ToppFun tool (ToppGene Suite) and three major pathway databases (Reactome, KEGG and PantherDB), pathway analyses were performed on the 221 genes confirming that neuronal-related pathways are highly prominent. Analysis of the Reactome database revealed 14/40 neuronal-related pathways (35.0\%). Higher percentage of nervous system-related pathways were observed in the KEGG (12/21 (57.1\%)) and PantherDB (10/11 (90.9\%)) pathway databases. More specifically, analysis using the Reactome database revealed pathways involved in synaptic protein interactions related to the following genes: SYT9, DLGAP3, NLGN4X, GRIN2A. Both the Reactome and KEGG databases identified pathways linked to neurotransmitter release with genes like RIMS1, SLC18A2 and SLC18A3 (Fig. 4b). Similarly, the PantherDB and the KEGG pathway databases analyses primarily identified pathways involved 

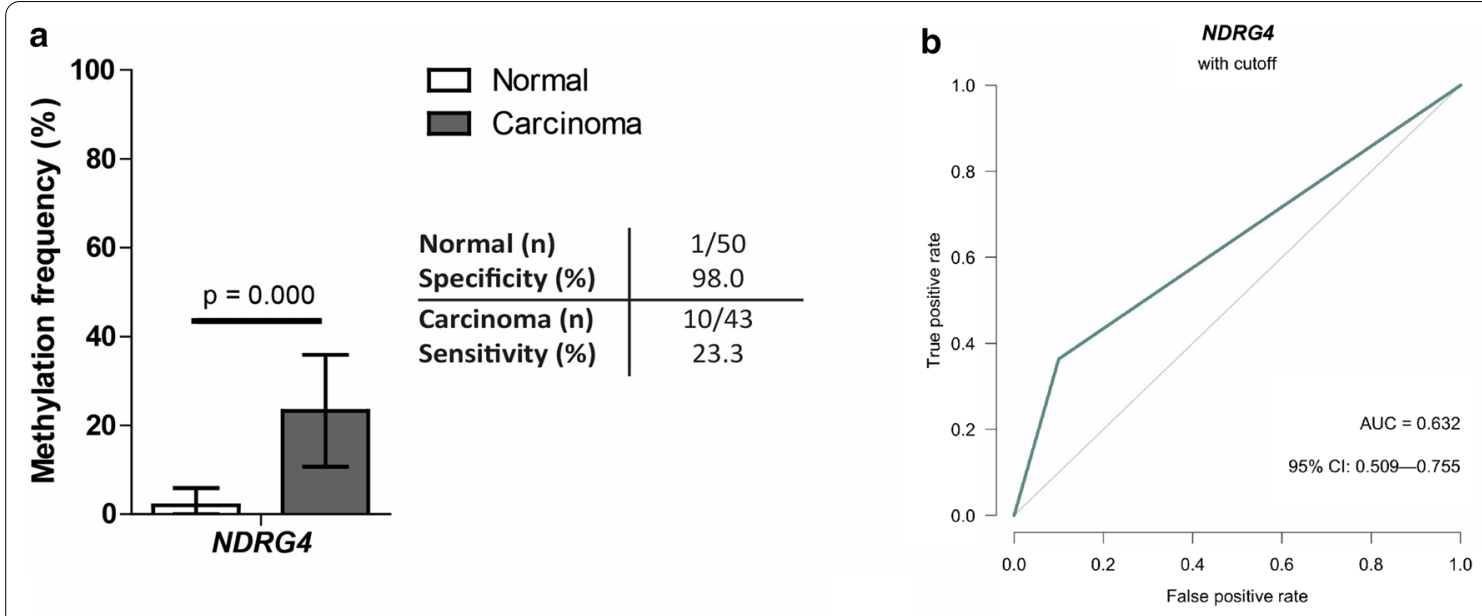

C
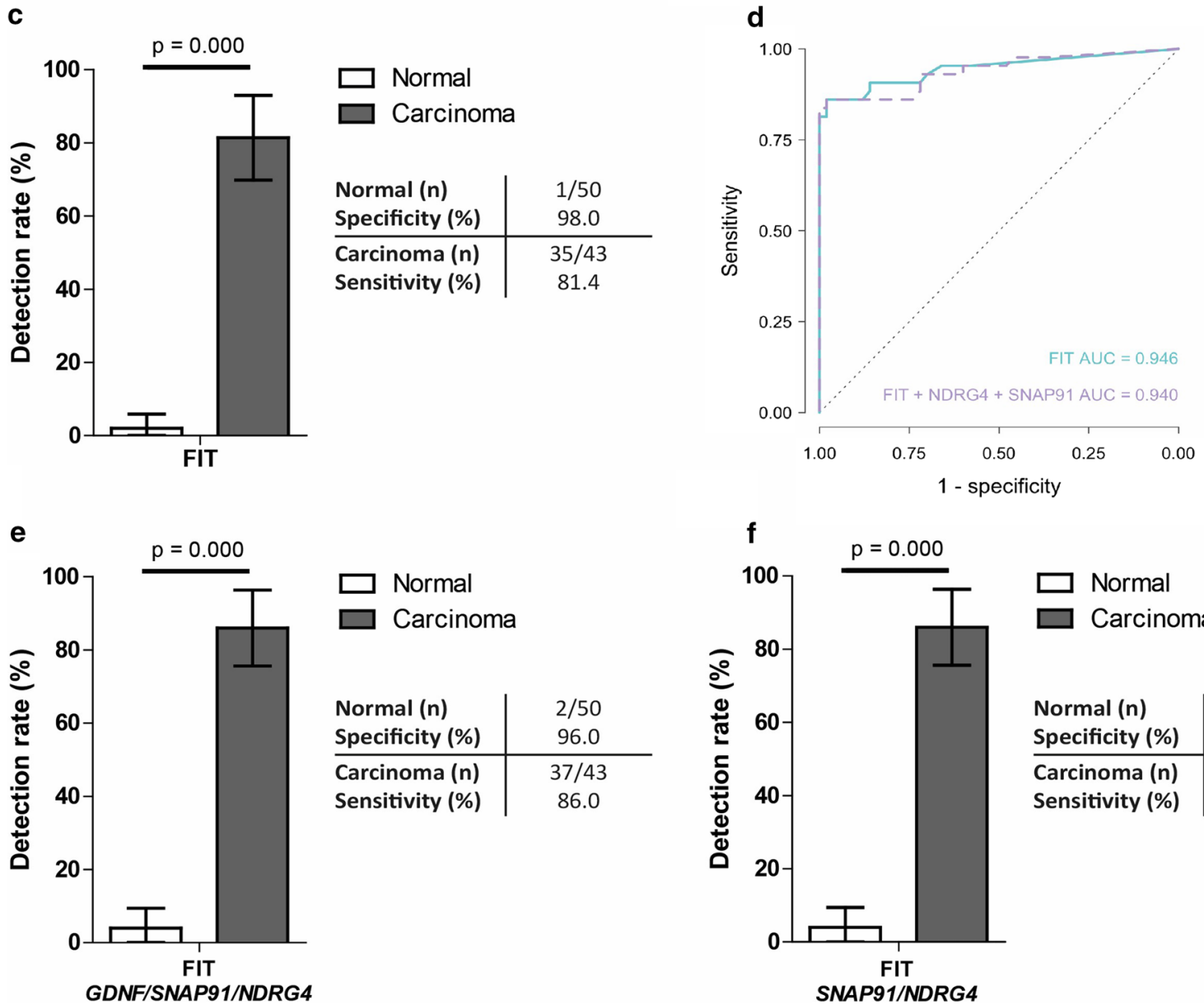

\begin{tabular}{l|c} 
Normal (n) & $2 / 50$ \\
Specificity (\%) & 96.0 \\
\hline Carcinoma (n) & $37 / 43$ \\
Sensitivity (\%) & 86.0
\end{tabular}

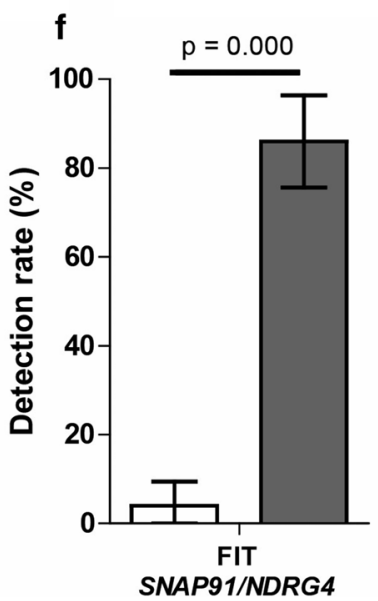

Fig. 3 NDRG4 methylation and FIT performance in fecal DNA and combined with the previously established marker panel. a ROC curve for NDRG4. The jagged (green) lines indicate the ROC curve. The gray line represents the line of no discrimination between good and bad classification. $\mathbf{b}$ Methylation frequency (\%) of NDRG4 methylation markers in fecal DNA of healthy controls (white bar) and carcinomas (dark gray bar). Pearson's chi-square test was used to calculate $P$ value. $\mathbf{c}$ The performance of FIT within the study population containing normal $(n=50)$ and carcinoma $(n=43)$ samples. $\mathbf{d}$ ROC curve for FIT alone and for FIT with GDNF/SNAP91/NDRG4. The green lines indicate the ROC curve for FIT alone while the purple line indicates the ROC for FIT/NDRG4/SNAP91. The gray line represents the line of no discrimination between good and bad classification. $e$, f The best performing marker panels GDNF/SNAP91/NDRG4 (e) and SNAP91/NDRG4 (f) in combination with FIT. For all figures, the $95 \% \mathrm{Cl}$ is shown with the error bars. Pearson's chi-square test was used to calculate $P$ values 


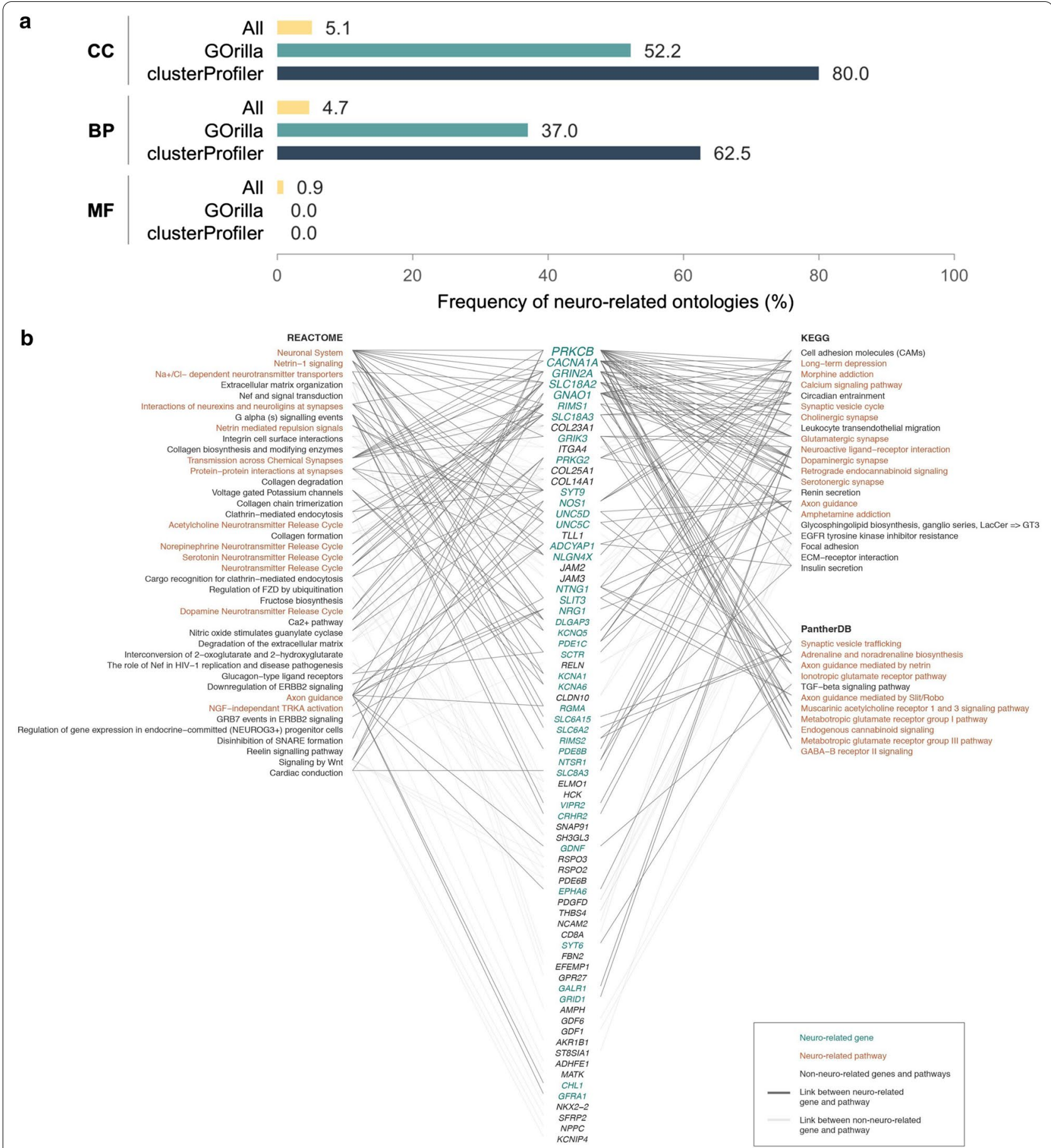

Fig. 4 Gene ontology enrichment and pathway analysis of the full set $(n=221)$ of identified methylation markers. a Comparisons of the frequency of neuro-related gene ontologies in the complete set of gene ontologies (yellow bar) versus the enriched sets obtained via analysis by GOrilla (green) and clusterProfiler (dark blue). The three major subsets of gene ontologies have been accounted for: cellular component (CC), biological process (BP) and molecular function (MF). b Pathway analysis was performed using the ToppGene tool based on 221 genes with potential as early detection biomarkers. ToppGene links, gene lists with pathways described in three major pathway databases (KEGG pathway, Reactome and PantherDB). Nervous system-related genes are highlighted in green, nervous system-related pathways in orange and genes and pathways with a neuronal background are linked with black lines, other with gray lines 
in neurotransmitter (receptor) signaling, particularly involving muscarinic acetylcholine, glutamate, GABA, serotonin and dopamine signaling (PRKCB, GNAO1, $C A C N A 1 A)$. Additionally, genes involved in synaptic vesicle trafficking were identified such as: RIMS1 and its family member RIMS2 next to SYT6, a family member of the aforementioned SYT9.

\section{Discussion}

Here, we used the publicly available TCGA data to identify novel DNA methylation markers for early, noninvasive detection of CRC [8]. All five identified methylation markers (GDNF, HAND2, SLC35F3, SNAP91 and SORCS1) were highly methylated in CRC samples but not in normal tissue. GDNF, HAND2, SNAP91 and SORCS1 have already been reported to be methylated in CRC tissue compared to normal tissue [30-33]. In stool samples from CRC patients, sensitivities decreased, ranging from $32.6 \%$ (HAND2) to $47.7 \%$ (SNAP91) at $98.0 \%$ specificity. This might be caused by the large amounts of bacterial DNA, multiple PCR-inhibiting substances such as polysaccharides, cell debris, proteins and bile salts in stool [34,35], and the small amounts of human DNA. The sensitivity could possibly be improved by using more sensitive methods for DNA methylation detection, such as the Discrimination of Rare EpiAlleles by Melt (DREAMing) technique [36] or digital PCR analyses [37].

Before biomarkers can be developed for use in clinical practice, comparison to the current golden standard is essential to draw conclusions on their diagnostic value [19, 20, 27, 38-40]. Combining our markers with FIT yielded a slightly improved CRC detection rate using a fixed specificity; however, this needs to be validated in larger populations. The addition of our previously identified marker, NDRG4, improved the performance of the stool-based panel. Future studies aiming to specifically identify DNA methylation markers complementary to NDRG4 using the publicly available TCGA data might even further improve the panel performance. Moreover, complementing our DNA methylation biomarkers with, for example, protein [41] or genetic [42] stool biomarkers could increase diagnostic performance. Finally, it should be considered that the performance of the DNA methylation markers in this study was based on the highest likelihood ratio, corresponding to $98.0 \%$ specificity. This was associated with lower sensitivities ranging from 32.9 to $46.5 \%$. However, within the research field of biomarker development, there is ample discussion on how the selection of cutoff values should be performed [43]. Although data-driven methods to maximize the diagnostic value of biomarkers are suggested, e.g., likelihood ratio and Youden index, decision-driven cutoff values matching a specific sensitivity or specificity are also used [43]. Using a predetermined cutoff matching a lower specificity could lead to an increase in sensitivity as the choice of cutoff is always a trade-off between sensitivity and specificity [43].

Among the in silico identified, potential diagnostic DNA methylation biomarkers, we found a significant enrichment of nervous system-related pathways and gene ontologies. This is in accordance with previous data $[31,44]$. Note that gene ontologies are structured as a hierarchical tree, meaning that different ontologies often share a significant number of genes and that they are therefore not independent. This impedes a statistical interpretation of the differences in the frequencies of neuro-related ontologies. Nevertheless, these results paint an intriguing picture of the potential role of the nervous system in CRC. Interestingly, all five of the markers assessed in this study are related to the nervous system, either by expression location, biological role or association with neurodegenerative disease. Previously, we observed that NDRG4, one of the Cologuard $^{\circledR}$ markers [27], is specifically expressed in nervous systems [28]. Although the role of the nervous system in CRC is understudied, several landmark papers have shown the importance of nerves in different types of cancer, promoting cancer growth, migration and invasion [32, 45-49]]. However, further studies are necessary to elucidate why the promoter regions of these neuronal genes are frequently methylated in CRC and whether this has biological consequences. Finally, further validation of our identified genes as CRC biomarkers is needed to investigate their added value to current screening tests. Moreover, their potential to detect early stage lesions, e.g., advanced adenomas, is yet to be studied.

\begin{abstract}
Abbreviations
AUC: Area under the curve; BMP3: Bone morphogenetic protein 3; BRCA : Breast cancer; Cl: Confidence interval; CRC: Colorectal cancer; DREAMing: Discrimination of Rare EpiAlleles by Melt; FDR: False-discovery rate; FFPE: Formalin-fixed paraffin embedded; FIT: Fecal immunochemical test; GDNF: Glial-derived neurotrophic factor; HAND2: Heart and neural crest derived 2; MSP: Methylation-specific PCR; NDRG4: N-myc downstream-regulated gene 4; PCR: Polymerase chain reaction; ROC: Receiver operating characteristics; SLC35F3: Solute carrier family 35 member F3; SNAP91: Synaptosome-associated protein 91; SORCS1: Sortilin-related VPS10 domain containing receptor 1; TCGA: The Cancer Genome Atlas.
\end{abstract}

\section{Supplementary Information}

The online version contains supplementary material available at https://doi. org/10.1186/s13148-021-01067-9.

Additional file 1. Supplementary methods and tables.

Additional file 2. Early detection methylation marker validation using carcinoma and matched normal tissue from CRC patients. 
Additional file 3. FIT and NDRG4 methylation performance in fecal DNA in combination with either the single markers or established marker panel.

\section{Acknowledgements}

This work was supported financially by KWF Kankerbestrijding grant (UM-213-6075) and by SU2C-DCS International Translational Cancer Research Dream Team Grant (Stand Up To Cancer (SU2C)-AACR- DT1415, MEDOCC). SU2C is a program of the Entertainment Industry Foundation administered by the American Association for Cancer Research.

\section{Declaration}

\section{Competing interests}

MvE is cofounder and shareholder of Epify BV, a molecular diagnostics company which develops DNA methylation markers for cancer diagnosis and personalized treatment.

\section{Author details}

1 Department of Pathology, GROW - School for Oncology and Developmental Biology, Maastricht University Medical Center, P.O. Box 616, 6200 MD Maastricht, The Netherlands. ${ }^{2}$ Department of Data Analysis and Mathematical Modelling, Ghent University, Ghent, Belgium. ${ }^{3}$ Department of Pathology, Netherlands Cancer Institute, Amsterdam, The Netherlands. ${ }^{4}$ The Hillman Cancer Center, University of Pittsburgh Cancer Institute, Pittsburgh, PA, USA. ${ }^{5}$ Department of Clinical Genetics, Erasmus University Medical Center, Rotterdam, The Netherlands.

\section{Received: 5 March 2021 Accepted: 4 April 2021}

\section{Published online: 15 April 2021}

\section{References}

1. Bray F, Ferlay J, Soerjomataram I, Siegel RL, Torre LA, Jemal A. Global cancer statistics 2018: GLOBOCAN estimates of incidence and mortality worldwide for 36 cancers in 185 countries. CA Cancer J Clin. 2018;68(6):394-424

2. Levin TR, Corley DA, Jensen CD, Schottinger JE, Quinn VP, Zauber AG, et al. Effects of organized colorectal cancer screening on cancer incidence and mortality in a large community-based population. Gastroenterology. 2018;155(5):1383-91.e5.

3. Schreuders EH, Ruco A, Rabeneck L, Schoen RE, Sung JJ, Young GP, et al. Colorectal cancer screening: a global overview of existing programmes. Gut. 2015;64(10):1637-49.

4. Navarro M, Nicolas A, Ferrandez A, Lanas A. Colorectal cancer population screening programs worldwide in 2016: an update. World J Gastroenterol. 2017;23(20):3632-42.

5. Quintero E, Castells A, Bujanda L, Cubiella J, Salas D, Lanas A, et al. Colonoscopy versus fecal immunochemical testing in colorectal-cancer screening. N Engl J Med. 2012;366(8):697-706.

6. Imperiale TF, Gruber RN, Stump TE, Emmett TW, Monahan PO. Performance characteristics of fecal immunochemical tests for colorectal cancer and advanced adenomatous polyps: a systematic review and meta-analysis. Ann Intern Med. 2019;170(5):319-29.

7. Dickinson BT, Kisiel J, Ahlquist DA, Grady WM. Molecular markers for colorectal cancer screening. Gut. 2015;64(9):1485-94.

8. Koch A, Joosten SC, Feng Z, de Ruijter TC, Draht MX, Melotte V, et al. Analysis of DNA methylation in cancer: location revisited. Nat Rev Clin Oncol. 2018;15(7):459-66.

9. Lamb YN, Dhillon S. Epi proColon((R)) 2.0 CE: a blood-based screening test for colorectal cancer. Mol Diagn Ther. 2017;21(2):225-32.

10. Jin P, Kang $Q$, Wang $X$, Yang L, Yu Y, Li N, et al. Performance of a secondgeneration methylated SEPT9 test in detecting colorectal neoplasm. J Gastroenterol Hepatol. 2015;30(5):830-3.

11. Toth K, Sipos F, Kalmar A, Patai AV, Wichmann B, Stoehr R, et al. Detection of methylated SEPT9 in plasma is a reliable screening method for both left- and right-sided colon cancers. PLoS ONE. 2012;7(9):e46000.
12. Johnson DA, Barclay RL, Mergener K, Weiss G, Konig T, Beck J, et al. Plasma Septin9 versus fecal immunochemical testing for colorectal cancer screening: a prospective multicenter study. PLoS ONE. 2014;9(6):e98238.

13. Potter NT, Hurban P, White MN, Whitlock KD, Lofton-Day CE, Tetzner $\mathrm{R}$, et al. Validation of a real-time PCR-based qualitative assay for the detection of methylated SEPT9 DNA in human plasma. Clin Chem. 2014;60(9):1183-91.

14. Tóth K, Wasserkort R, Sipos F, Kalmár A, Wichmann B, Leiszter K, et al. Detection of methylated septin 9 in tissue and plasma of colorectal patients with neoplasia and the relationship to the amount of circulating cell-free DNA. PLoS ONE. 2014;9(12):e115415-e.

15. Ørntoft M-BW, Nielsen HJ, Ørntoft TF, Andersen CL, Danish Study Group on Early Detection of Colorectal C. Performance of the colorectal cancer screening marker Sept9 is influenced by age, diabetes and arthritis: a nested case-control study. BMC Cancer. 2015;15:819.

16. Song L, Li Y, Jia J, Zhou G, Wang J, Kang Q, et al. Algorithm optimization in methylation detection with multiple RT-qPCR. PLOS ONE. 2016;11(11):e0163333.

17. Song L, Jia J, Peng X, Xiao W, Li Y. The performance of the SEPT9 gene methylation assay and a comparison with other CRC screening tests: a meta-analysis. Sci Rep. 2017;7(1):3032.

18. Gyparaki MT, Basdra EK, Papavassiliou AG. DNA methylation biomarkers as diagnostic and prognostic tools in colorectal cancer. J Mol Med (Berl). 2013;91(11):1249-56.

19. Imperiale TF, Ransohoff DF, Itzkowitz SH, Levin TR, Lavin P, Lidgard GP, et al. Multitarget stool DNA testing for colorectal-cancer screening. N Engl J Med. 2014;370(14):1287-97.

20. Ahlquist DA, Zou H, Domanico M, Mahoney DW, Yab TC, Taylor WR, et al. Next-generation stool DNA test accurately detects colorectal cancer and large adenomas. Gastroenterology. 2012;142(2):248-56 (quiz e25-6).

21. Bosch LJW, Melotte V, Mongera S, Daenen KLJ, Coupé VMH, van Turenhout ST, et al. Multitarget stool DNA test performance in an average-risk colorectal cancer screening population. Am J Gastroenterol. 2019;114(12):1909-18.

22. Wrangle J, Machida EO, Danilova L, Hulbert A, Franco N, Zhang W, et al. Functional identification of cancer-specific methylation of CDO1, HOXA9, and TAC1 for the diagnosis of lung cancer. Clin Cancer Res. 2014;20(7):1856-64.

23. van Lanschot MCJ, Carvalho B, Coupé VMH, van Engeland M, Dekker E, Meijer GA. Molecular stool testing as an alternative for surveillance colonoscopy: a cross-sectional cohort study. BMC Cancer. 2017;17(1):116.

24. Eden E, Navon R, Steinfeld I, Lipson D, Yakhini Z. GOrilla: a tool for discovery and visualization of enriched $\mathrm{GO}$ terms in ranked gene lists. BMC Bioinform. 2009;10(1):48.

25. Yu G, Wang L-G, Han Y, He Q-Y. clusterProfiler: an R Package for comparing biological themes among gene clusters. OMICS J Integr Biol. 2012;16(5):284-7

26. Chen J, Bardes EE, Aronow BJ, Jegga AG. ToppGene Suite for gene list enrichment analysis and candidate gene prioritization. Nucleic Acids Res. 2009;37(suppl_2):W305-11.

27. Melotte $\mathrm{V}$, Lentjes $\mathrm{MH}$, van den Bosch SM, Hellebrekers DM, de Hoon JP, Wouters KA, et al. N-Myc downstream-regulated gene 4 (NDRG4): a candidate tumor suppressor gene and potential biomarker for colorectal cancer. J Natl Cancer Inst. 2009;101(13):916-27.

28. Vaes N, Lentjes M, Gijbels MJ, Rademakers G, Daenen KL, Boesmans W, et al. NDRG4, an early detection marker for colorectal cancer, is specifically expressed in enteric neurons. Neurogastroenterol Motil. 2017;29(9):e13095.

29. Schonkeren SL, Massen M, van der Horst R, Koch A, Vaes N, Melotte V. Nervous NDRGs: the N-myc downstream-regulated gene family in the central and peripheral nervous system. Neurogenetics. 2019;20:173-86.

30. Sambuudash $\mathrm{O}, \mathrm{Kim}$ HS, Cho MY. Lack of aberrant methylation in an adjacent area of left-sided colorectal cancer. Yonsei Med J. 2017;58(4):749-55.

31. Yang Y, Chu FH, Xu WR, Sun JQ, Sun X, Ma XM, et al. Identification of regulatory role of DNA methylation in colon cancer gene expression via systematic bioinformatics analysis. Medicine (Baltimore). 2017;96(47):e8487.

32. Hua Y, Ma X, Liu X, Yuan X, Qin H, Zhang X. Abnormal expression of mRNA, microRNA alteration and aberrant DNA methylation patterns in rectal adenocarcinoma. PLoS ONE. 2017;12(3):e0174461.

33. Beggs A, James J, Leong K, Caldwell G, Ronlund K, Bach S, et al. Hypermethylation of SNAP91 as an alternative mechanism of epidermal 
growth factor signalling dysregulation: a genome-wide meta-analysis with validation of colorectal cancers. Lancet. 2014;383:S25.

34. Mirsepasi H, Persson S, Struve C, Andersen LO, Petersen AM, Krogfelt KA. Microbial diversity in fecal samples depends on DNA extraction method: easyMag DNA extraction compared to QIAamp DNA stool mini kit extraction. BMC Res Notes. 2014;7:50.

35. Roperch J-P, Benzekri K, Mansour H, Incitti R. Improved amplification efficiency on stool samples by addition of spermidine and its use for noninvasive detection of colorectal cancer. BMC Biotechnol. 2015:15:41.

36. Pisanic TR 2nd, Athamanolap P, Poh W, Chen C, Hulbert A, Brock MV, et al. DREAMing: a simple and ultrasensitive method for assessing intratumor epigenetic heterogeneity directly from liquid biopsies. Nucleic Acids Res. 2015:43(22):e154.

37. Liu Y, Wu H, Zhou Q, Song Q, Rui J, Zou B, et al. Digital quantification of gene methylation in stool DNA by emulsion-PCR coupled with hydrogel immobilized bead-array. Biosens Bioelectron. 2017;92:596-601.

38. Lu H, Huang S, Zhang X, Wang D, Zhang X, Yuan X, et al. DNA methylation analysis of SFRP2, GATA4/5, NDRG4 and VIM for the detection of colorectal cancer in fecal DNA. Oncol Lett. 2014;8(4):1751-6.

39. Park SK, Baek HL, Yu J, Kim JY, Yang HJ, Jung YS, et al. Is methylation analysis of SFRP2, TFPI2, NDRG4, and BMP3 promoters suitable for colorectal cancer screening in the Korean population? Intest Res. 2017;15(4):495-501.

40. Xiao W, Zhao H, Dong W, Li Q, Zhu J, Li G, et al. Quantitative detection of methylated NDRG4 gene as a candidate biomarker for diagnosis of colorectal cancer. Oncol Lett. 2015;9(3):1383-7.

41. Komor MA, Bosch LJ, Coupé VM, Rausch C, Pham TV, Piersma SR, et al. Proteins in stool as biomarkers for non-invasive detection of colorectal adenomas with high risk of progression. J Pathol. 2020;250(3):288-98.
42. Kanthan R, Senger J-L, Kanthan SC. Fecal molecular markers for colorectal cancer screening. Gastroenterol Res Pract. 2012;2012:184343.

43. Vetter TR, Schober P, Mascha EJ. Diagnostic testing and decisionmaking: beauty is not just in the eye of the beholder. Anesth Analg. 2018;127(4):1085-91.

44. Fadda A, Gentilini D, Moi L, Barault L, Leoni VP, Sulas P, et al. Colorectal cancer early methylation alterations affect the crosstalk between cell and surrounding environment, tracing a biomarker signature specific for this tumor. Int J Cancer. 2018;143(4):907-20.

45. Rademakers G, Vaes N, Schonkeren S, Koch A, Sharkey KA, Melotte V. The role of enteric neurons in the development and progression of colorectal cancer. Biochim Biophys Acta Rev Cancer. 2017;1868(2):420-34.

46. Magnon C, Hall SJ, Lin J, Xue X, Gerber L, Freedland SJ, et al. Autonomic nerve development contributes to prostate cancer progression. Science. 2013;341(6142):1236361.

47. Zhao CM, Hayakawa Y, Kodama Y, Muthupalani S, Westphalen CB, Andersen GT, et al. Denervation suppresses gastric tumorigenesis. Sci Transl Med. 2014:6(250):250.

48. Hayakawa Y, Sakitani K, Konishi M, Asfaha S, Niikura R, Tomita H, et al. Nerve growth factor promotes gastric tumorigenesis through aberrant cholinergic signaling. Cancer Cell. 2017;31(1):21-34.

49. Stopczynski RE, Normolle DP, Hartman DJ, Ying H, DeBerry JJ, Bielefeldt K, et al. Neuroplastic changes occur early in the development of pancreatic ductal adenocarcinoma. Can Res. 2014;74(6):1718-27.

\section{Publisher's Note}

Springer Nature remains neutral with regard to jurisdictional claims in published maps and institutional affiliations.
Ready to submit your research? Choose BMC and benefit from:

- fast, convenient online submission

- thorough peer review by experienced researchers in your field

- rapid publication on acceptance

- support for research data, including large and complex data types

- gold Open Access which fosters wider collaboration and increased citations

- maximum visibility for your research: over $100 \mathrm{M}$ website views per year

At BMC, research is always in progress.

Learn more biomedcentral.com/submissions 